\title{
Illumina/Nanopore reads encoding $\beta$-lactamases from plasmids (synthetic vectors) in patient sequencing data from China and Hong-Kong in the suspected coronavirus driven COVID19 outbreak
}

Sandeep Chakraborty

\section{Letter}

I have hypothesized based on sequencing data - two from China [1,2], and one from Hong-Kong [3] - that the SARS-CoV-2 has integrated in the Prevotella genome $[4,4,5]$. The only fly in the ointment in this theory is chimeric reads arising from $16 \mathrm{~S}$ integrations [6]. These chimeric reads in that region, although some of the are within proteins (SI.plasmid:chemeric.inprotein.fa).

Apart from this, this hypothesis (a chimeric bacteria/virus) explains many of the intriguing observations - the extremely high false negatives (this is now DNA, while we are looking for RNA) [7,8], high incubation periods [9], abdominal problems presenting before respiratory problems [10]. All these, taken one-by-one, probably happen in many viral diseases. But the combination of all observations strongly indicates this is a bacteria+virus.

\section{Plasmid reads encoding $\beta$-lactamases}

Here, I report plasmid reads encoding $\beta$-lactamases in patient sequencing data from China [2] and HongKong [3]. It is not there in the other Chinese study [1].

It is a distant possibility that these reads are from bacteria, given the high homology to plasmids (Fig 1). The origin of these reads, very unlikely to be contamination, in two different geographical locations needs serious investigation. It might also help in choosing the anti-biotics being prescribed.

These sequences can be obtained from SI.plasmid/Study2.HK.blase.fa (N=62) and SI.plasmid/Study3.China.blase.fa $(\mathrm{N}=35)$.

\section{References}

1. Wu F, Zhao S, Yu B, Chen Y, Wang W, et al. (2020) Complete genome characterisation of a novel coronavirus associated with severe human respiratory disease in Wuhan. China bioRxiv 24.

2. Chen L, Liu W, Zhang Q, Xu K, Ye G, et al. (2020) RNA based mNGS approach identifies a novel human coronavirus from two individual pneumonia cases in 2019 Wuhan outbreak. Emerging Microbes \& Infections 9: 313-319.

3. Chan JFW, Yuan S, Kok KH, To KKW, Chu H, et al. (2020) A familial cluster of pneumonia associated with the 2019 novel coronavirus indicating person-to-person transmission: a study of a family cluster. The Lancet .

4. Chakraborty S (2020). The 2019 Wuhan outbreak could be caused by the bacteria prevotella, which is aided by the coronavirus, possibly to adhere to epithelial cells - prevotella is present in huge amounts in patients from both China and Hong Kong. doi:10.31219/osf.io/usztn. URL osf .io/usztn.

5. Chakraborty S (2020). The Wuhan coronavirus has integrated in Prevotella, which possibly causes the observed extreme virulence - as sequencing data from 2 different studies in China and Hong-Kong shows unequivocally. doi:10.31219/osf.io/ktngw. URL osf .io/ktngw. 
6. Haas BJ, Gevers D, Earl AM, Feldgarden M, Ward DV, et al. (2011) Chimeric 16s rrna sequence formation and detection in sanger and 454-pyrosequenced pcr amplicons. Genome research 21: 494504 .

7. Hegarty $\mathrm{S}$ The Chinese doctor who tried to warn others about coronavirus https://www.bbc.com/news/amp/world-asia-china-51364382]. BBC, 2020 .

8. Chakraborty S (2020). Significant false negatives in RT-PCR detection of Wuhan coronavirus happens as the usually RNA-stranded bacteria is now DNA within the prevotella genome - and we are looking for RNA, which will be made in lesser amount by the bacteria. doi:10.31219/osf.io/85ypb. URL osf.io/85ypb.

9. Lauer SA, Grantz KH, Bi Q, Jones FK, Zheng Q, et al. (2020) The incubation period of 2019-ncov from publicly reported confirmed cases: estimation and application. medRxiv .

10. Wang D, Hu B, Hu C, Zhu F, Liu X, et al. (2020) Clinical characteristics of 138 hospitalized patients with 2019 novel coronavirus-infected pneumonia in Wuhan, China. JAMA .

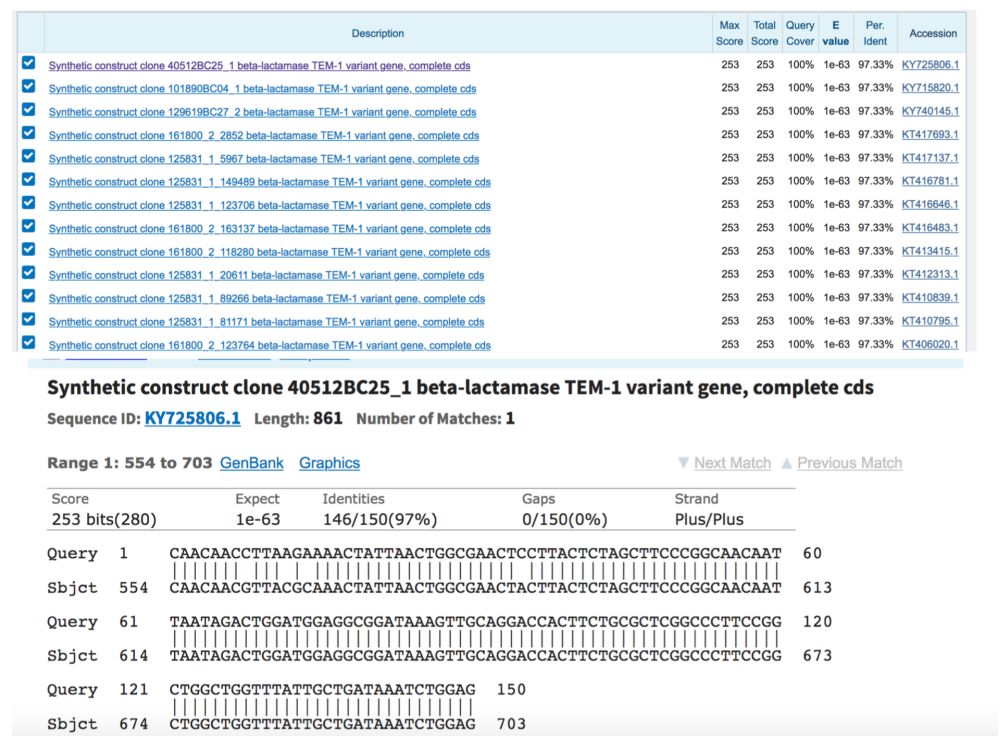

Figure 1: Reads matching to $\beta$-lactmase encoding plasmids: Plasmid reads encoding $\beta$-lactamases in patient sequencing data from China [2] and Hong-Kong [3] in the COVID19 outbreak. It is not there in other Chinese study [1]. 